\title{
Analytical Techniques for the Doppler Signature of Sea Surfaces in the Microwave Regime-II: Nonlinear Surfaces
}

\author{
Frédéric Nouguier ${ }^{1,{ }^{*}}$, Charles-Antoine Guérin ${ }^{2}$, Gabriel Soriano $^{3}$
}

\author{
${ }^{1}$ IFREMER, Lab Oceanog Spatiale, F-29280 Plouzane, France \\ 2 Univ Sud Toulon Var, Lab Sondages Electromagnet Environm Terr, Geosci \& Remote Sensing Dept, \\ Ctr Natl Rech Sci,Unite Mixte Rech 6017, F-83957 La Garde, France \\ ${ }_{3}^{3}$ Univ Aix Marseille 3, Inst Fresnel, Ctr Natl Rech Sci, Unite Mixte Rech 6133, F-13013 Marseille, \\ France
}

*: Corresponding author : Frédéric Nouguier, email address : frederic.nouguier@gmail.com

\begin{abstract}
:
This paper extends the results of a previous work by combining hydrodynamic and electromagnetic analytical models for the simulation of the ocean Doppler spectrum at microwave frequencies. We consider weakly nonlinear sea surfaces after the choppy wave model and incorporate them in classical and unified scattering models, namely, the Kirchhoff and weighted curvature approximations. We show that statistical expressions can be obtained for the Doppler spectrum in a way similar to the case of linear surfaces. As expected, the nonlinear nature of the sea surface dramatically impacts the Doppler spectrum at moderate to large incidence angles, with a shift of the central frequency and a broadening of the spectrum. Monte Carlo comparisons are performed with the Creamer model, which is frequently used to describe weakly nonlinear sea surfaces but does not enjoy a statistical formulation for the Doppler characteristics. The same qualitative behavior is found but some quantitative differences are found and discussed.
\end{abstract}

Keywords : Doppler spectrum; microwave; nonlinear gravity waves; remote sensing; rough surfaces; scattering; sea surface 


\section{Introduction}

In a previous paper [1] the authors developed technique to obtain the main Doppler characteristics of the sea surface in the microwave regime in the framework of asymptotic scattering models. The aim of the present work is to combine these results with recent nonlinear models for the sea surface. It is wellknown that hydrodynamic nonlinearities have a dramatic impact on the Doppler signature, especially at large incidence, where they are responsible for the shift and broadening of the central peak. However the inclusion of sea surface nonlinearities in a scattering model is a challenge which has found only few partial solutions. The classical approach to tackle the problem relies on the scattering Two-Scale Model ([2]) and the inclusion of surface nonlinearities is realized through a Modulation Tranfert Function [3] or bound capillary waves [4]. In the last decade, a series of papers presented numerical simulations of sea Doppler spectra ([5], [6], [7], [8], [9], [10]) with rigorous scattering formulation and weakly nonlinear hydrodynamics after Creamer [11] and West models [12]. As no statistical formulation was available, Doppler spectra had to be gathered by averaging the backscattered _eld from a large number of time-evolving sample surfaces, a procedure which is highly time-consuming even with todays facilities. A further difficulty arises from the Creamer technique [11], which remains computationnaly demanding and actually dissuasive for twodimensional (2D) surfaces. On the other hand West technique is numerically more e_cient but has been abandonned in view of stability issues. Therefore most of the studies have been limited to one-dimensional (1D) surfaces, with the exception of [13], [14], which were restricted to L-band and small wind speeds, however.

Numerical runs on 1D surfaces have been found qualitatively consistent with experimental observations, inasmuch as they also evidence an increase of the central frequency and a broadening of the spectra. To simulate realistic scenes in view of geophysical applications, efficient and accurate models are needed. In this respect, the potential of constantly improving asymptotic models has not been fully exploited. In particular the recent Choppy Wave Model [15], [16] has proven to enjoy some desirable properties such as analytical simplicity and numerical effciency. Note that the use of this model in the context of Doppler calculations is not entirely new, as a similar Lagrangian model has been introduced in [17] in combination 
with the so-called generalized curvature expansion [18]. This promising approach, however, does not seem to have been pursued.

We will show that the analytical approach which was developed for linear surfaces can be fully adapted to the case of choppy surfaces, yielding efficient statistical formulation of the first two moments of the Doppler spectrum. As before, the theory will be developped for fully 2D surfaces and numerically compared with the reference test cases of Toporkov and Brown [5] according to the Creamer model. Even though the Choppy Wave Model fulfils the same qualitative expectations as the Creamer model, there are still some noticeable differences which will be discussed.

\section{The Choppy Wave Model}

Recently some of the authors [15] proposed a weakly nonlinear model termed "Choppy Wave Model" (CWM) based on a Lagrangian description of sea surface particles. The CWM can be constructed in a simple way by horizontal displacement of a linear surface. Consider a linear time-dependent surfaces in the form:

$$
\eta(\mathbf{r}, t)=\int_{\mathbb{R}^{2}} d \mathbf{k}\left[a(\mathbf{k}) \mathrm{e}^{-\mathrm{i} \omega_{\mathbf{k}} t}+a^{*}(-\mathbf{k}) \mathrm{e}^{\mathrm{i} \omega_{\mathbf{k}} t}\right] \mathrm{e}^{\mathrm{i} \mathbf{k} \cdot \mathbf{r}}
$$

where $a(\mathbf{k})$ is the complex amplitude of the wave, $\mathbf{k}$ the associated wave number and $\omega_{\mathbf{k}}=\sqrt{g|\mathbf{k}|\left(1+|\mathbf{k}|^{2} / k_{M}^{2}\right)}$ is the gravity-capillarity dispersion relationship $\left(k_{M}=363.2 \mathrm{rad} . \mathrm{m}^{-1}\right.$ is the wave number with minimum phase speed). The CWM is obtained by performing the following transformation:

$$
(\mathbf{r}, \eta(\mathbf{r}, t)) \rightarrow(\mathbf{r}+\mathbf{D}(\mathbf{r}, t), \eta(\mathbf{r}, t))
$$

with $\mathbf{D}$ the so-called Riesz transform of the linear surface $\eta$ :

$$
\mathbf{D}(\mathbf{r}, t)=\int_{\mathbb{R}^{2}} d \mathbf{k} \mathbf{i} \widehat{\mathbf{k}}\left[a(\mathbf{k}) \mathrm{e}^{-\mathrm{i} \omega_{\mathbf{k}} t}+a^{*}(-\mathbf{k}) \mathrm{e}^{\mathrm{i} \omega_{\mathbf{k}} t}\right] \mathrm{e}^{\mathrm{i} \mathbf{k} \cdot \mathbf{r}},
$$

where $\widehat{\mathbf{k}}=\mathbf{k} /|\mathbf{k}|$ and $a(\mathbf{k})$ is the complex amplitude of the wave defined by its wave number $\mathbf{k}$. The corresponding nonlinear surface, $\tilde{\eta}$, is implicitely defined by the relation:

$$
\tilde{\eta}(\mathbf{r}+\mathbf{D}(\mathbf{r}, t), t)=\eta(\mathbf{r}, t)
$$

Henceforth, the tilde superscript will address quantities pertaining to the nonlinear surface (for example $\tilde{\eta}, \tilde{C}(t), \tilde{S}(t)$, etc). The model is numerically efficient as it can be entirely generated by Fast Fourier Transform. Stricly speaking, the CWM is not fully consistent with Hamiltonian expansion techniques [11] and the classical perturbative approach [19] as it has a small vorticity and is not complete at second-order of steepness, a default which is the price to pay for numerical efficiency. However, the CWM was compared a posteriori with the Creamer and Longuet-Higgins solution and found to be close, especially for narrow spectra. It captures some, but not all, the features of the fully nonlinear solution, such as the vertical asymmetry of elevations and the kurtosis of slopes. In addition it provides analytical expressions for some statistical descriptors of nonlinear gravity waves in infinite depth. Later on, the model was found very 
useful in the framework of remote sensing applications [20] as it makes it possible to obtain statistical expressions for the incoherent NRCS and, consequently, does not require Monte Carlo averaging over a large number of sample surfaces. Note, however, that CWM is a weakly nonlinear model and is not able to describe near-breaking crests. Therefore breaking events are clearly not taken into account in this model. In this section, we adapt the CWM to nonlinear moving surfaces for the purpose of Doppler spectrum calculation. In the CWM framework, time evolution of nonlinear surface is driven by the underlying linear surface which, together with its Riesz transform, is calculable at any time without knowledge of the past. Thus, time dependency is included in formulae derived by [15] and [20] without loss of generality and with much simplicity.

Even through the nonlinear effect of the CWM on the NRCS was found rather small, a strong impact is observed on the Doppler signal. Indeed, the CWM corrects the horizontal component of particles velocities by a displacement related to elevations. In such a process, long waves strongly affect the shorter ones which are "dopplerized" by the corresponding horizontal displacement. Doppler spectrum depends on these velocities and a modulation of Bragg waves by longer waves therefore impacts its shape. Since the Doppler velocity is obtained by projection on the line-of-sight of the radar, we expect a greater impact of the CWM at high incidence angles in the upwind direction.

\section{Kirchhoff Approximation for the Choppy Wave Model}

The scattering amplitude from a nonlinear CWM surface can be easily obtained under the Kirchhoff Approximation (KA) and will henceforth be designated as KA-CWM. For the geometry and definition of the scattering problem we adopt the same notational conventions as in [1]. Taking eq. (8) from [20] and adding time dependency the KA-CWM scattering amplitude can be written:

$$
\tilde{\mathbb{S}}_{K A}(t)=\mathbb{K} \frac{1}{(2 \pi)^{2}} \int_{\mathbb{R}^{2}} d \mathbf{r} \mathrm{e}^{\mathrm{i} \mathbf{Q}_{\mathbf{H}} \cdot \mathbf{r}} \mathrm{e}^{\mathrm{i} Q_{z} \eta(\mathbf{r}, t)+\mathrm{i} \mathbf{Q}_{\mathbf{H}} \cdot \mathbf{D}(\mathbf{r}, t)} J(\mathbf{r}, t),
$$

where $J$ is the Jacobian of the transformation $\mathbf{r} \rightarrow \mathbf{r}+\mathbf{D}(\mathbf{r}, t)$ defined by

$$
J(\mathbf{r}, t)=\left|\begin{array}{cc}
1+\partial_{x} D_{x}(\mathbf{r}, t) & \partial_{x} D_{y}(\mathbf{r}, t) \\
\partial_{y} D_{x}(\mathbf{r}, t) & 1+\partial_{y} D_{y}(\mathbf{r}, t)
\end{array}\right|
$$

Accounting for time dependence in the calculations detailed in [20] leads to :

$$
\tilde{\mathcal{C}}_{K A}(t)=\frac{|\mathbb{K}|^{2}}{Q_{z}^{2}} \frac{1}{\pi} \int_{\mathbb{R}^{2}} d \mathbf{r} \mathrm{e}^{\mathrm{i} \mathbf{Q}_{\mathbf{H}} \cdot \mathbf{r}}\left[\mathrm{e}^{-Q_{z}^{2}(\rho(\mathbf{0}, 0)-\rho(\mathbf{r}, t))} \tilde{\mathcal{F}}(\mathbf{r}, t)-\mathrm{e}^{-Q_{z}^{2} \rho(\mathbf{0}, 0)} \tilde{\mathcal{F}}(\infty, t)\right]
$$

with

$\tilde{\mathcal{F}}(\mathbf{r}, t)=\exp \left(-Q_{H}^{2}\left(\rho_{\widehat{\mathbf{Q}}_{H}}(\mathbf{0}, 0)-\rho_{\widehat{\mathbf{Q}}_{H}}(\mathbf{r}, t)\right)\right)\left(\left[1-\mathrm{i} \mathbf{Q}_{\mathbf{H}} \cdot \nabla \rho(\mathbf{r}, t)\right]^{2}-\Delta \rho(\mathbf{r}, t)+Q_{z}^{2}\left(\rho_{1}(\mathbf{0}, 0)-\rho_{1}(\mathbf{r}, t)\right)^{2}\right)$ 
where $\rho, \nabla \rho$ and $\Delta \rho$ are respectively the covariance function, its Gradient and its Laplacian and where the auxiliary functions $\rho_{\widehat{\mathbf{Q}}_{H}}$ and $\rho_{1}$ are defined by:

$$
\begin{aligned}
\rho_{\widehat{\mathbf{Q}}_{H}}(\mathbf{r}, t) & =\int_{\mathbb{R}^{2}} d \mathbf{k}\left(\widehat{\mathbf{Q}}_{H} \cdot \widehat{\mathbf{k}}\right)^{2}\left[a(\mathbf{k}) \mathrm{e}^{-\mathrm{i} \omega_{\mathbf{k}} t}+a^{*}(-\mathbf{k}) \mathrm{e}^{\mathrm{i} \omega_{\mathbf{k}} t}\right] \mathrm{e}^{\mathrm{i} \mathbf{k} \cdot \mathbf{r}} \\
\rho_{1}(\mathbf{r}, t) & =\int_{\mathbb{R}^{2}} d \mathbf{k}|\mathbf{k}|\left[a(\mathbf{k}) \mathrm{e}^{-\mathrm{i} \omega_{\mathbf{k}} t}+a^{*}(-\mathbf{k}) \mathrm{e}^{\mathrm{i} \omega_{\mathbf{k}} t}\right] \mathrm{e}^{\mathrm{i} \mathbf{k} \cdot \mathbf{r}} .
\end{aligned}
$$

As pointed out by [20] the nonlinear surface constructed with the CWM has a modified spectrum (termed "dressed") with respect to the underlying linear surface (whose spectrum is referred to as "undressed"). However at small winds the dressed and undressed spectra are very close and we will not distinguish them. We thus used the same spectrum for both linear and nonlinear surfaces in 1D calculations. Again we chosed the Pierson-Moskovitz (PM) spectrum for comparison purposes with earlier works, even though it is not the most realistic one.

Figure 1 shows the Doppler spectra in the KA for linear and CWM surfaces at $5 \mathrm{~m} \cdot \mathrm{s}^{-1}$ wind speed, for a PM spectrum at 50 degrees incidence angle in $\mathrm{X}$ band (electromagnetic wavelength $\lambda_{e}=3 \mathrm{~cm}$ ). It has been obtained from the Fourier transform of equation (III.6) in a few minutes on an ordinary personal computer. The numerical efficiency of the CWM provides the alternative possibility to evaluated Doppler spectra through a Monte-Carlo averaging over a certain number of deterministic time-evolving sample surfaces, as it is usually done in the case of rigorous electromagnetic solutions [5]. This is useful to verify the statistical formulation of the Doppler spectra or to make comparisons with other hydrodynamic models for which there is no such statistical formulation (for instance the Creamer model [11], see section V). On Figure 1 we have superimposed the Monte-Carlo results over one-hundred linear and nonlinear surfaces, showing an excellent agreement with the statistical formulation. The results obtained under the Weighted Curvature Approximation in horizontal polarization and its associated Monte-Carlo spectrum are also superimposed for further comparisons.

The analytical formulations of the Doppler central frequency $f_{c}$ and width $\gamma$ in the CWM framework can be easily derived with the same technique as in the linear case [1]. Here, however, it has been found more convenient to derive them from the Fourier transform of equation (III.6). Figures 2 and 3 show $f_{c}$ and $\gamma$ versus angle incidence in $\mathrm{X}$ band at 5, 7 and $9 \mathrm{~m} \cdot \mathrm{s}^{-1}$ wind speed for linear and CWM surfaces under the KA. As expected, higher Doppler frequencies are observed when passing to nonlinear surfaces, the increase being more pronounced at high winds. The free Bragg frequency is no longer recovered at large incidence angles. An even more dramatic impact is observed on the Doppler width, which is found much larger than in the linear case and quasi-insensitive to the incidence angles above 40 degrees while the linear counterpart falls off rapidly. The elevated value reached in the nonlinear case strongly increases with wind, pointing out that the hydrodynamic modulation highly affects the speed variance of sensed waves. 


\section{Weighted Curvature Approximation for the Choppy Wave Model}

The most complete approach, from both electromagnetic and hydrodynamic points of view, is the combination of the Weighted Curvature Approximation (WCA, [21]) and the CWM (referred to as WCACWM). This reaches a high level of analytical complexity (even through the numerical efficiency is almost the same as a mere KA) and we will therefore again restrict to 1D surfaces. We found it advantageous to start with the formulation of WCA based on square slopes rather than second derivatives:

$$
\tilde{\mathbb{S}}_{W C A}(t)=\mathbb{B} \frac{1}{2 \pi} \int_{\mathbb{R}} d x \mathrm{e}^{\mathrm{i} Q_{H} x} \mathrm{e}^{\mathrm{i} Q_{z} \tilde{\eta}(x, t)}-\frac{Q_{z}}{Q_{H}^{2}} \mathbb{T} \frac{1}{2 \pi} \int_{\mathbb{R}} d x \tilde{\eta}^{\prime}(x, t)^{2} \mathrm{e}^{\mathrm{i} Q_{H} x} \mathrm{e}^{\mathrm{i} Q_{z} \tilde{\eta}(x, t)},
$$

where $\tilde{\eta}$ is the nonlinear surface, $\tilde{\eta}^{\prime}$ its derivative with respect to the horizontal variable $x$ and $\mathbb{T}=$ $\mathbb{B}-\mathbb{K}$ is the difference between the Bragg and Kirchhoff kernel. Now differentiating the implicit relation $\tilde{\eta}(x+D(x, t), t)=\eta(x, t)$ leads to:

$$
\tilde{\eta}^{\prime}(x+D(x, t), t)=\frac{\eta^{\prime}(x, t)}{\left(1+D^{\prime}(x, t)\right)} .
$$

Explicit integrand can be obtained by operating the change of variable $x \rightarrow x+D(x, t)$, yielding:

$$
\tilde{\mathbb{S}}_{W C A}(t)=\mathbb{B} \frac{1}{2 \pi} \int_{\mathbb{R}} d x\left(1+D^{\prime}(x, t)\right) \mathrm{e}^{\mathrm{i} Q_{H} x} \mathrm{e}^{\mathrm{i} Q_{z} \eta(x, t)}-\frac{Q_{z} \mathbb{T}}{Q_{H}^{2}} \frac{1}{2 \pi} \int_{\mathbb{R}} d x \frac{\eta^{\prime}(x, t)^{2}}{1+D^{\prime}(x, t)} \mathrm{e}^{\mathrm{i} Q_{H} x} \mathrm{e}^{\mathrm{i} Q_{z} \eta(x, t)} .
$$

Note that the quantities involved in this integration now pertain to the linear surface and its derivatives $\left(\eta, \eta^{\prime}, D, D^{\prime}\right)$ and are thus random Gaussian processes. The calculation of the associated field covariance function or NRCS involves the two-point characteristic function and some associated functions:

$$
\left\langle\zeta(x, t) \mathrm{e}^{\mathrm{i} Q_{z}(\eta(x, t)-\eta(0, t))}\right\rangle,
$$

where $\zeta(x, t)$ stands for a set of functions related to $\eta^{\prime}$ and $D^{\prime}$. Some of these calculations are classical, some other are not and have been relegated to the Appendix. After lenghty calculations we obtain the following formula for the WCA-CWM field covariance function:

$$
\begin{aligned}
\tilde{C}_{W C A}(t) & =\tilde{C}_{S S A}(t)+\frac{Q_{z}^{2}}{Q_{H}^{4}} \int_{\mathbb{R}} \mathrm{e}^{\mathrm{i} Q_{H} x} d x\left[\tilde{\mathcal{F}}_{2}(x, t) \mathrm{e}^{-Q_{z}^{2} S_{0} / 2}-\tilde{\mathcal{F}}_{2}(\infty, t) \mathrm{e}^{-Q_{z}^{2} \rho(0,0)}\right] \\
& -\frac{\mathrm{i}}{Q_{H}^{2}} \int_{\mathbb{R}} \mathrm{e}^{\mathrm{i} Q_{H} x} d x\left[\tilde{\mathcal{F}}_{1}(x, t) \mathrm{e}^{-Q_{z}^{2} S_{0} / 2}-\tilde{\mathcal{F}}_{1}(\infty, t) \mathrm{e}^{-Q_{z}^{2} \rho(0,0)}\right]
\end{aligned}
$$

where the expressions of the functions $\tilde{\mathcal{F}}_{1 / 2}$ are given in the Appendix. Note that $\tilde{C}_{S S A}$ is the onedimensional counterpart of equation (III.6) of $\tilde{C}_{K A}$, with the Kirchhoff kernel $\mathbb{K}$ replaced by the Bragg kernel $\mathbb{B}$.

As before, this statistical expression can be checked with the solution obtained through a Monte-Carlo procedure. The scattering amplitude (IV.12) has been computed over one-hundred time-evolving surfaces and compared with the results of the (IV.14). Figure 4 shows the Doppler central frequency as a function of the incidence angle for the WCA-CWM in X band at $5 \mathrm{~m} \cdot \mathrm{s}^{-1}$ wind speed. Here again, the statistical WCA CWM curves have been derived from the incidence angle dependent Fourier transform of equation 
(IV.14). The Bragg frequency is still given as reference. The statistical formulation is in overal good agreement with the Monte-Carlo averaging, except at large incidences in horizontal polarization, and much less computationally time consuming. The noticeable differences (greater than 10\%) in horizontal polarization at grazing angles are a measure of the computations accuracy. For the Monte Carlo process, the averaging error decreases as the inverse square root of the number of sample surfaces. At grazing angles, the Monte Carlo technique is very sensitive to the size and shape of the tapered beam which is used to illuminate the surfaces while the statistical formulation corresponds to an incident plane wave. However, the statistical formulation suffers from numerical difficulties at high incidence angles in horizontal polarization. The reason is that the WCA NRCS in horizontal polarization is the small difference of two larger positive terms of same order of magnitude and thus requires more accuracy on the latter than the V-pol counterpart, which writes as the sum of two positive terms. Hence the field correlation function and consequently the Doppler spectrum are more difficult to evaluate. As shown on figure 5, in both polarizations, the Doppler widths under WCA-CWM are extremely close to the one obtained in the KA case and are in very good agreement with the Monte-Carlo averaging on the whole range of incidence angles. For next section comparison purpose, we have superimposed, on figures 4 and 5, a more detailed and accurate version of the statistical results presented in [5]. As discussed in the next section, the nonlinear hydrodynamic model used in their sea surface generation (Creamer model) is responsible of the observed discrepancies.

\section{COMPARISON WITH OTHER NONLINEAR MODELS}

There are only few theoretical models in the literature for the Doppler return of nonlinear sea surfaces in the microwave regime. One approach is based on the Two-scale model [2] combined with the Modulation Transfer Function (MTF) technique [3], which allows to take into account the modulation of short waves by larger waves ("hydrodynamical" MTF) as well as the influence of facet tilting on the cross-section ("tilt" MTF). This approach provides simple expressions for the mean and width of the Doppler spectrum as weighted averages of the sea elevation spectrum (eq. (10) and (11) of [3]). The corresponding values have been shown to be consistent, at least qualitatively, with experimental results [3] and other numerical techniques [6]. However, its main shortcoming is the rather heuristic derivation of the MTF. Another, increasingly popular, approach is the Creamer nonlinear model for sea surface [11], which is based on a vertical deformation of an underlying linear surface. Even through the resulting expression for the nonlinear surface is quite simple, the model is not numerically efficient as no Fast Fourier Transform can be used as in the CWM. The generation of a one-dimensional surface with $N$ sampling points requires typically $\sim N^{2}$ operations contrarily to the CWM which is $\sim N \log (N)$. Hence Monte-Carlo averaging over Creamer surfaces implies a massive computational burden even with today facilities.

Contrarily to the MTF or the CWM approach, the Creamer model does not provide statistical expressions for the Doppler characteristics. To make a fair comparison with our results we have used the 
Monte-Carlo rather than the statistical technique to calculate Doppler spectra in the WCA-CWM model. To exclude differences originating from the electromagnetic method we also computed the WCA applied to deterministic Creamer surfaces (WCA-Creamer). Figure 6 and 7 show the evolution of $f_{c}$ with incidence according to WCA-CWM and WCA-Creamer for each polarization. Even if the same numerical electromagnetic method is employed for the two hydrodynamic models, noticeable differences in both vertical and horizontal polarizations remain on the most part of the incidence range of variation, showing that CWM and Creamer model are not equivalent for remote sensing purposes. To understand this difference we investigated the distribution of slopes and curvatures according to both models. Figure 8 clearly shows that the Creamer and CWM lead to slightly different slope distributions and strongly different curvature distributions, the occurence of positive curvatures being much higher in the case of the Creamer model. Note that these distributions are obtained from sample surfaces with a high-frequency cut-off on the elevation spectrum $\left(k_{\max }=137 \mathrm{rad} \cdot \mathrm{m}^{-1}\right.$ on the figure) set at five times the electromagnetic wavenumber. Results of [5] are superimposed on figures 6 and 7. The agreement between the central Doppler frequency predicted by MOMI-Creamer and WCA-Creamer is excellent in vertical polarization (less than 1\% error) and remains within $10 \%$ relative accuracy in horizontal polarization, except at grazing angles. This shows that the WCA model is reliable at non-grazing angles, even for nonlinear surfaces which exhibit sharper crests. The problem of grazing angles, already known in NRCS calculations, is still an issue for Doppler calculation with asymptotic methods. Results from the MTF approach [3], actually taken from [6], are also given. A mere piecewise curve regression was used to fit their data. They compare well with the MOMI-Creamer model between 20 and 60 degrees incidence. At grazing angles the MOMI-Creamer model has an explosive behavior which cannot be predicted by any of the analytical models. The conclusion is that the Creamer model is actually more strongly nonlinear than CWM inasmuch as it exhibits stronger slopes and curvatures and produces enhanced Doppler shifts.

Another well-known nonlinear surface model is the West model [12]. It is based on an efficient operator expansion and is in principle capable to include arbitrary orders of nonlinearity. In practice it suffers from stability issues due to the explosion of high frequencies which must be therefore filtered. In view of these numerical issues we did not test this model. However, numerical comparisons performed in earlier works [7], [8] show that Doppler spectra calculate according to this model exhibit even stronger nonlinear features than the Creamer model, with broader spectra and the occurence of an additional peak at the negative Bragg frequency indicating reverse travelling waves (which are ignored by assumption in our model).

\section{Conclusion}

This paper has investigated the characteristics of the microwave sea Doppler spectrum in the framework of analytical electromagnetical and hydrodynamical models. We have combined the CWM with the KA and WCA analytical scattering model and obtained statistical expressions for the field correlation function 
which prevent from running highly demanding Monte-Carlo simluations. The most complete model is the combination of WCA and CWM, which is sensitive to both polarization and hydrodynamical modulation and still enjoys a statistical, numerically efficient, formulation. A comparaison has been made with the extensive numerical experiments by Toporkov and Brown, with a rigorous electromagnetic model (MoMI) and the Creamer hydrodynamic model. The WCA-CWM model displays the expected qualitative features of the Doppler return, namely an enhanced Doppler shift in horizontal polarization and a saturated Doppler width as the incidence increases. Some discrepancies have been found with the MoMI-Creamer model. To find out the origin of these discrepancies, the WCA-Creamer model has also been implemented and found in excellent agreement with the MoMI-Creamer model for all but grazing incidents. This shows that differences can be attributed to the hydrodynamic models in consideration. An inspection of the slopes and curvatures distribution actually reveals that the Creamer model and CWM are not equivalent in this respect as the former predicts stronger positive curvatures than the latter. While the WCA scattering model seems to be reliable now, the sensibility to the nonlinear surface model shows the necessity to pursue the research on the hydrodynamical issues.

Acknowledgments. This work was supported by the ANR Project No. ANR-09-BLAN-0232-01 SIMODE. Many thanks go to D.R. Thompson for helpful discussion and to J.V. Toporkov for providing us supplementary data.

\section{REFERENCES}

[1] F. Nouguier, C.A. Guérin, and Soriano G. Analytical techniques for the doppler signature of sea surfaces in the microwave regime. i: Linear surfaces. submitted.

[2] DR Thompson. Calculation of microwave Doppler spectra from the ocean surface with a time-dependent composite model. Kluwer Academic Publishers, 1989.

[3] R. Romeiser and D.R. Thompson. Numerical study on the along-track interferometric radar imaging mechanism of oceanic surface currents. Geoscience and Remote Sensing, IEEE Transactions on, 38(1):446-458, 2000.

[4] V.U. Zavorotny and A.G. Voronovich. Two-scale model and ocean radar Doppler spectra at moderate-and low-grazing angles. Antennas and Propagation, IEEE Transactions on, 46(1):84-92, 1998.

[5] J. V. Toporkov and G. S. Brown. Numerical simulations of scattering from time-varying, randomly rough surfaces. IEEE Trans. Geophys. Remote Sensing, 38:1616-1624, July 2000.

[6] J.V. Toporkov, M.A. Sletten, and G.S. Brown. Numerical scattering simulations from time-evolving ocean-like surfaces at L-and X-band: Doppler analysis and comparisons with a composite surface analytical model. Proc. of XXVII General Assembly of URSI, 2002.

[7] J.T. Johnson, J.V. Toporkov, and G.S. Brown. A numerical study of backscattering from time-evolving sea surfaces: comparison of hydrodynamic models. Geoscience and Remote Sensing, IEEE Transactions on, 39(11):2411-2420, 2001.

[8] A.R. Hayslip, J.T. Johnson, and G.R. Baker. Further numerical studies of backscattering from time-evolving nonlinear sea surfaces. Geoscience and Remote Sensing, IEEE Transactions on, 41(10):2287-2293, 2003.

[9] JV Toporkov and MA Sletten. Statistical properties of low-grazing range-resolved sea surface backscatter generated through two-dimensional direct numerical simulations. IEEE Transactions on Geoscience and Remote Sensing, 45(5 Part 1):1181-1197, 2007.

[10] J.T. Johnson, R.J. Burkholder, J.V. Toporkov, D.R. Lyzenga, and W.J. Plant. A Numerical Study of the Retrieval of 
Sea Surface Height Profiles From Low Grazing Angle Radar Data. Geoscience and Remote Sensing, IEEE Transactions on, 47(6):1641-1650, 2009.

[11] D. B. Creamer, F. Henyey, R. Schult, and J. Wright. Improved linear representation of ocean surface waves. J. Fluid Mech., 205:135-161, 1989.

[12] B. West and al. A new numerical method for surface hydrodynamics. J. Geophys. Res., C11:803-824, 1987.

[13] G. Soriano, M. Joelson, and M. Saillard. Doppler spectra from a two-dimensional ocean surface at L-band. Geoscience and Remote Sensing, IEEE Transactions on, 44(9):2430-2437, 2006.

[14] X. Li and X. Xu. Scattering and Doppler Spectral Analysis for Two-Dimensional Linear and Nonlinear Sea Surfaces. Geoscience and Remote Sensing, IEEE Transactions on, 49(2):603-611, 2011.

[15] F. Nouguier, C.A. Guérin, and B. Chapron. Choppy wave model for nonlinear gravity waves. Journal of Geophysical Research (JGR)-Oceans, 114(C09012):1-16, 2009.

[16] F. Nouguier, C.A. Guérin, and B. Chapron. Scattering From Nonlinear Gravity Waves: The 'Choppy Wave' Model. Geoscience and Remote Sensing, IEEE Transactions on, 48(12):4184-4192, 2010.

[17] IF Pedersen, G. Engen, and H. Johnsen. Polarization Dependency in Sea Surface Doppler Frequency Shift and its Application to Envisat ASAR Alt-Pol Data. In Envisat E ERS Symposium, volume 572, page 41, 2005.

[18] G. Engen, I. Friestad-Pedersen, H. Johnsen, and T. Elfouhaily. Curvature effects in ocean surface scattering. IEEE Trans. Antennas Propag., 54(5):1370-1379, 2006.

[19] MS Longuet-Higgins. The effect of non-linearities on statistical distributions in the theory of sea waves. Journal of Fluid Mechanics, 17(03):459-480, 1963.

[20] F. Nouguier, C.A. Guérin, and B. Chapron. Scattering from non-linear gravity waves: the "choppy wave" model. Geoscience and Remote Sensing, IEEE Transactions on, 2010.

[21] C.A. Guérin, G. Soriano, and B. Chapron. The Weighted Curvature Approximation in scattering from sea surfaces. Waves in Random and Complex Media, 20(3):364-384, 2010.

\section{APPENDIX}

\section{SOME NONTRIVIAL CORRElators}

Some nontrivial statistical expressions are required for the calculations involved in the WCA-CWM model. The technique relies as usual on the calculation of n-points characteristic functions of Gaussian process and their derivatives. As usual the braket symbols $\langle$.$\rangle means the statistical average over realisa-$ tions. The prime superscript means the space derivation and the "0" subscript means that quantities are taken at space and time origins.

$$
\begin{aligned}
\left\langle\frac{\eta_{0}^{\prime 2}\left(1+D^{\prime}\right)}{\left(1+D_{0}^{\prime}\right)} E\right\rangle & =\mathrm{i} \operatorname{erfc1}\left(\frac{1}{\sqrt{2 \sigma_{2}^{2}}}\right)\left[-2 \rho_{1}^{\prime} f_{+}+\left(g_{+}+\mathrm{i}\right)\left(f_{+}^{2}-\sigma_{2}^{2}\right)\right] \mathcal{E} \\
\left\langle\frac{\eta^{\prime 2}\left(1+D_{0}^{\prime}\right)}{\left(1+D^{\prime}\right)} E\right\rangle & =\mathrm{i} \operatorname{erfc1}\left(\frac{1}{\sqrt{2 \sigma_{2}^{2}}}\right)\left[-2 \rho_{1}^{\prime} f_{-}+\left(g_{-}+\mathrm{i}\right)\left(f_{-}^{2}-\sigma_{2}^{2}\right)\right] \mathcal{E} \\
\left\langle\frac{\eta^{\prime 2} \eta_{0}^{\prime 2}}{\left(1+D^{\prime}\right)\left(1+D_{0}^{\prime}\right)} E\right\rangle & =\left[2 \rho^{\prime \prime}\left(\rho^{\prime \prime}-2 f_{+} f_{-}\right)+\left(f_{-}^{2}-\sigma_{2}^{2}\right)\left(f_{+}^{2}-\sigma_{2}^{2}\right)\right] \operatorname{erfc} 2\left(\rho^{\prime \prime}\right) \mathcal{E}
\end{aligned}
$$


with

$$
\begin{aligned}
E & =\exp \left[\mathrm{i} Q_{H}\left(D-D_{0}\right)+\mathrm{i} Q_{z}\left(\eta-\eta_{0}\right)\right] \\
\mathcal{E} & =\exp \left[-\left(Q_{H}^{2}+Q_{z}^{2}\right) S_{0} / 2\right] \\
f_{ \pm} & =Q_{H} S_{1} / 2 \pm Q_{z} \rho^{\prime} \\
g_{ \pm} & =Q_{H} \rho^{\prime} \pm Q_{z} S_{1} / 2 \\
\rho_{1} & =\int|k| \Gamma(k) \mathrm{e}^{\mathrm{i} k x-\mathrm{i} \operatorname{sgn}(k) \omega_{k} t} d k \\
S_{0} & =2\left(\rho_{0}-\rho(x, t)\right) \\
S_{1} & \left.=2\left(\rho_{1}\right)_{0}-\rho_{1}(x, t)\right) \\
\operatorname{erfc} 1(z) & =z \sqrt{\pi} \mathrm{e}^{z^{2}} \operatorname{erfc}(z) \\
\operatorname{erfc} 2\left(\rho^{\prime \prime}\right) & =2 \int_{0}^{\pi / 4}\left(-\rho_{0}^{\prime \prime}-\rho^{\prime \prime} \sin (2 \theta)\right)^{-1}\left[1-\operatorname{erfc} 1\left(\sqrt{\frac{1+\sin (2 \theta)}{2\left(-\rho_{0}^{\prime \prime}-\rho^{\prime \prime} \sin (2 \theta)\right)}}\right)\right] d \theta
\end{aligned}
$$

where erfc is the well known complementary error function. For numerical purpose, we can note that the erfc2 function can easily be tabuled and that $\operatorname{erfc} 2(0)=\operatorname{erfc}^{2}\left(1 / \sqrt{2 \sigma_{2}^{2}}\right)$.

In WCA-CWM statistical equation (IV.14), the integrands are

$$
\begin{aligned}
\tilde{\mathcal{F}}_{1}= & \operatorname{erfc1}\left(\frac{1}{\sqrt{2 \sigma_{2}^{2}}}\right) \mathrm{e}^{-Q_{H}^{2} S_{0} / 2} \times \\
& \left\{\mathbb{B} \mathbb{T}^{*}\left[-2 \rho_{1}^{\prime} f_{+}+\left(g_{+}+\mathrm{i}\right)\left(f_{+}^{2}-\sigma_{2}^{2}\right)\right]+\mathbb{B}^{*} \mathbb{T}\left[-2 \rho_{1}^{\prime} f_{-}+\left(g_{-}+\mathrm{i}\right)\left(f_{-}^{2}-\sigma_{2}^{2}\right)\right]\right\} \\
\tilde{\mathcal{F}}_{2}= & |\mathbb{T}|^{2}\left[2 \rho^{\prime \prime}\left(\rho^{\prime \prime}-2 f_{+} f_{-}\right)+\left(f_{-}^{2}-\sigma_{2}^{2}\right)\left(f_{+}^{2}-\sigma_{2}^{2}\right)\right] \operatorname{erfc} 2\left(\rho^{\prime \prime}\right) \mathrm{e}^{-Q_{H}^{2} S_{0} / 2}
\end{aligned}
$$

where $\mathbb{T}=\mathbb{B}-\mathbb{K}$. 


\section{List OF FigurES}

1 Comparison of statistical and Monte-Carlo KA and KA-CWM Doppler spectrum shape at 50 degree incidence in $\mathrm{X}$ band with $U_{19.5}=5 \mathrm{~m} . \mathrm{s}^{-1}$. WCA results in horizontal polarization are also superimposed. . . . . . . . . . . . . . . . . . . . . . . 112

2 Comparison of KA and KA-CWM Doppler central frequency in X band with $U_{19.5}=5,7,9$ m.s ${ }^{-1} .112$

3 Comparison of KA and KA-CWM Doppler width in X band with $U_{19.5}=5,7,9$ m.s ${ }^{-1}$. . 113

4 Comparison of Monte-Carlo and Statistical WCA-CWM Doppler central frequency in, X band with $U_{19.5}=5 \mathrm{~m} . \mathrm{s}^{-1}$. T\&B : Toporkov \& Brown results obtained with the exact electromagnetic Method of Order Multiple Interactions and the Creamer model. . . . . . . . 113

5 Comparison of Monte-Carlo and Statistical WCA-CWM Doppler width in X band with $U_{19.5}=5 \mathrm{~m} . \mathrm{s}^{-1}$. T\&B : Toporkov \& Brown results obtained with the exact electromagnetic Method of Order Multiple Interactions and the Creamer model. . . . . . . . . . . . . . 114

6 Comparison of Monte-Carlo CWM and Creamer WCA Doppler central frequency in L band, vertical polarization with $U_{19.5}=5 \mathrm{~m} . \mathrm{s}^{-1}$. T\&B : Toporkov \& Brown results based on the MOMI and Creamer model. Thompson-Romeiser results are based on a TSM coupled with

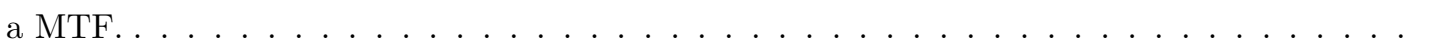

7 Comparison of Monte-Carlo CWM and Creamer WCA Doppler central frequency in L band, horizontal polarization with $U_{19.5}=5 \mathrm{~m} . \mathrm{s}^{-1}$. T\&B : Toporkov \& Brown results based on the MOMI and Creamer model. Thompson-Romeiser results are based on a TSM coupled with

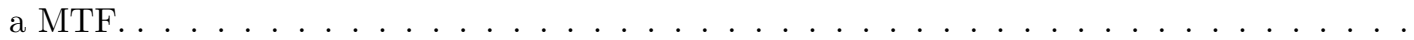

8 Probability distribution functions with a truncated Pierson Moskovitz spectrum at $k_{\max }=$ 137 rad.m ${ }^{-1}, U_{19.5}=5$ m.s ${ }^{-1} \ldots \ldots \ldots \ldots \ldots \ldots \ldots \ldots \ldots$ 


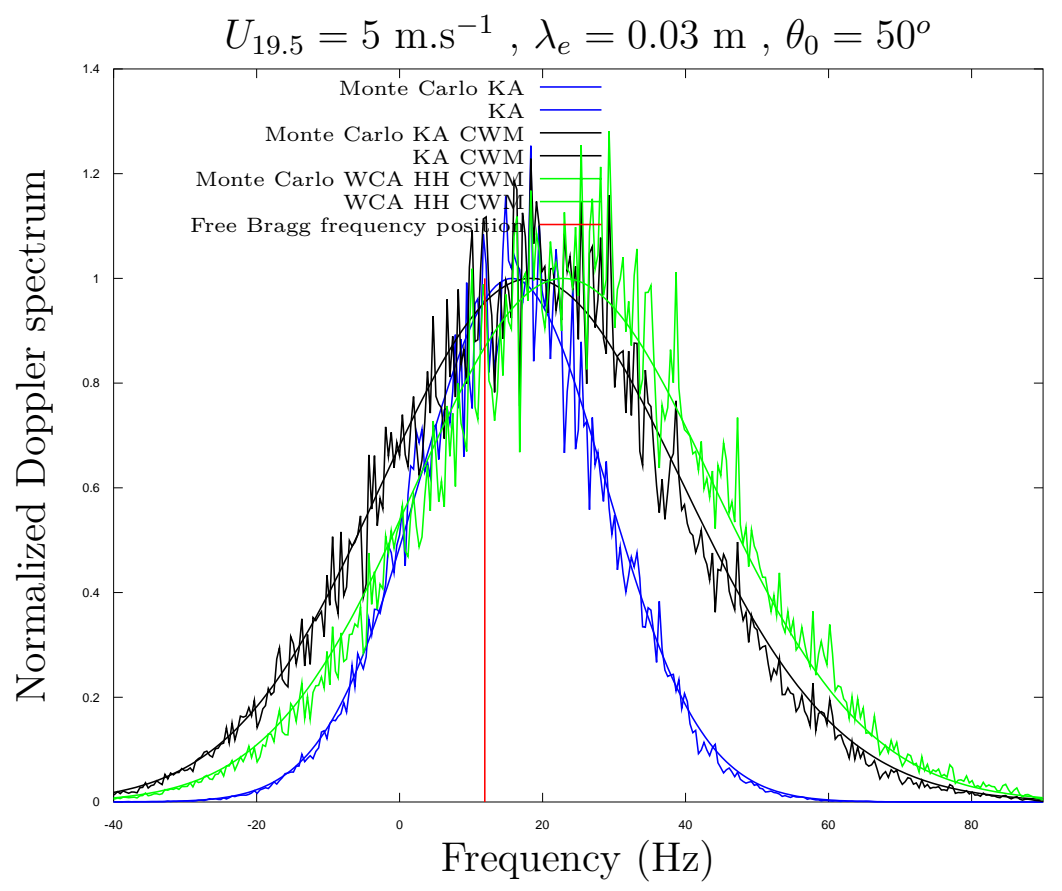

Fig. 1. Comparison of statistical and Monte-Carlo KA and KA-CWM Doppler spectrum shape at 50 degree incidence in X band with $U_{19.5}=5 \mathrm{~m} . \mathrm{s}^{-1}$. WCA results in horizontal polarization are also superimposed.

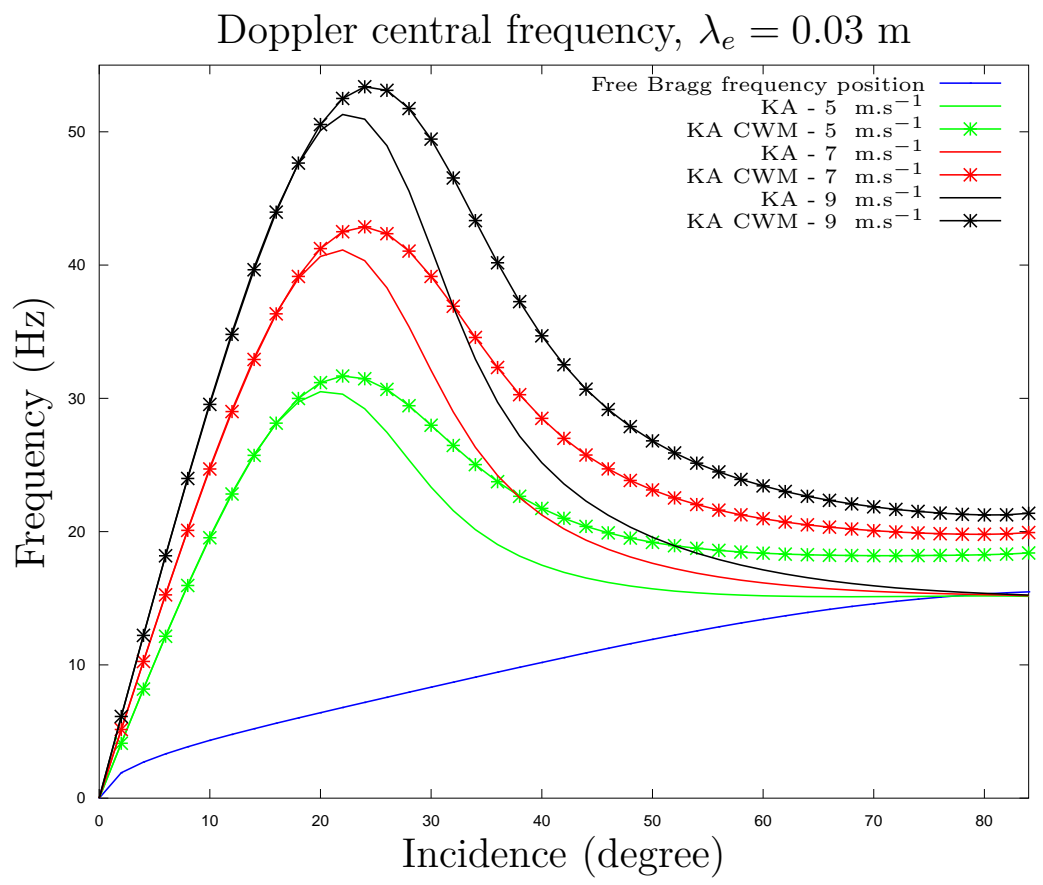

Fig. 2. Comparison of KA and KA-CWM Doppler central frequency in X band with $U_{19.5}=5,7,9$ m.s ${ }^{-1}$. 


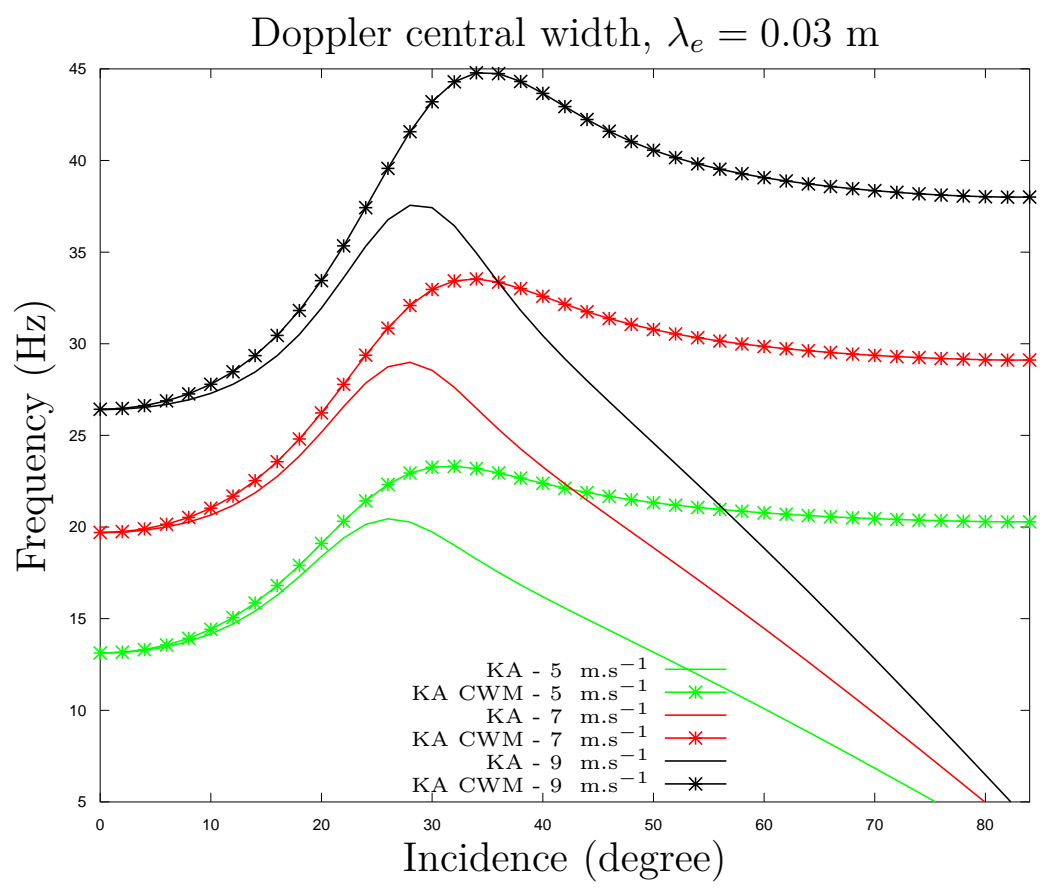

Fig. 3. Comparison of KA and KA-CWM Doppler width in X band with $U_{19.5}=5,7,9 \mathrm{~m} . \mathrm{s}^{-1}$.

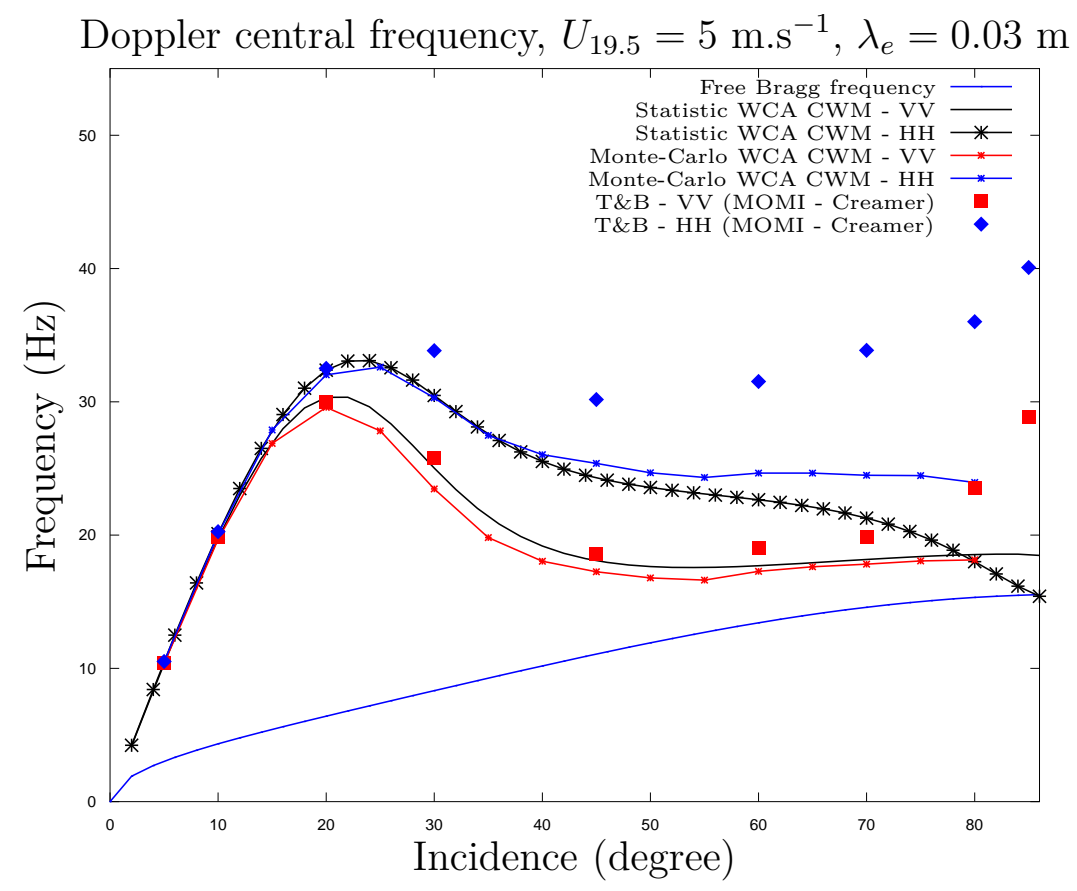

Fig. 4. Comparison of Monte-Carlo and Statistical WCA-CWM Doppler central frequency in, X band with $U_{19.5}=5 \mathrm{~m} . \mathrm{s}^{-1}$. T\&B : Toporkov \& Brown results obtained with the exact electromagnetic Method of Order Multiple Interactions and the Creamer model. 


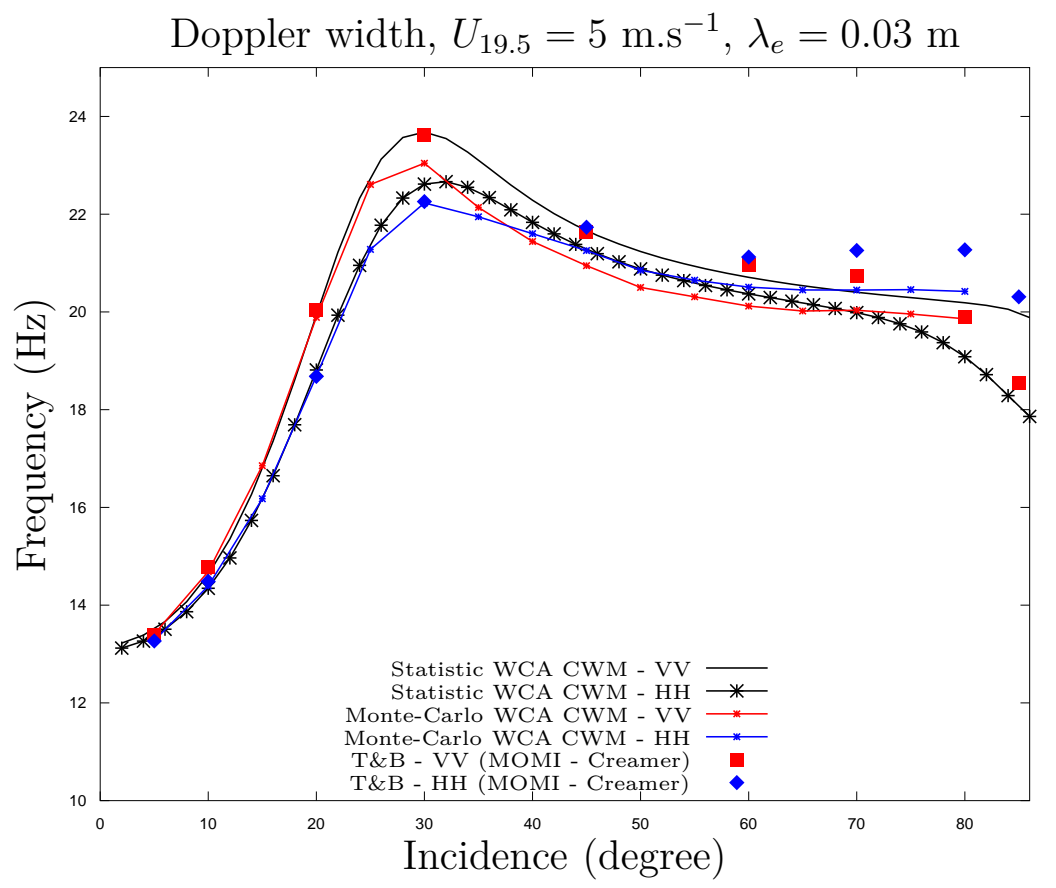

Fig. 5. Comparison of Monte-Carlo and Statistical WCA-CWM Doppler width in X band with $U_{19.5}=5 \mathrm{~m} . \mathrm{s}^{-1}$. T\&B : Toporkov \& Brown results obtained with the exact electromagnetic Method of Order Multiple Interactions and the Creamer model.

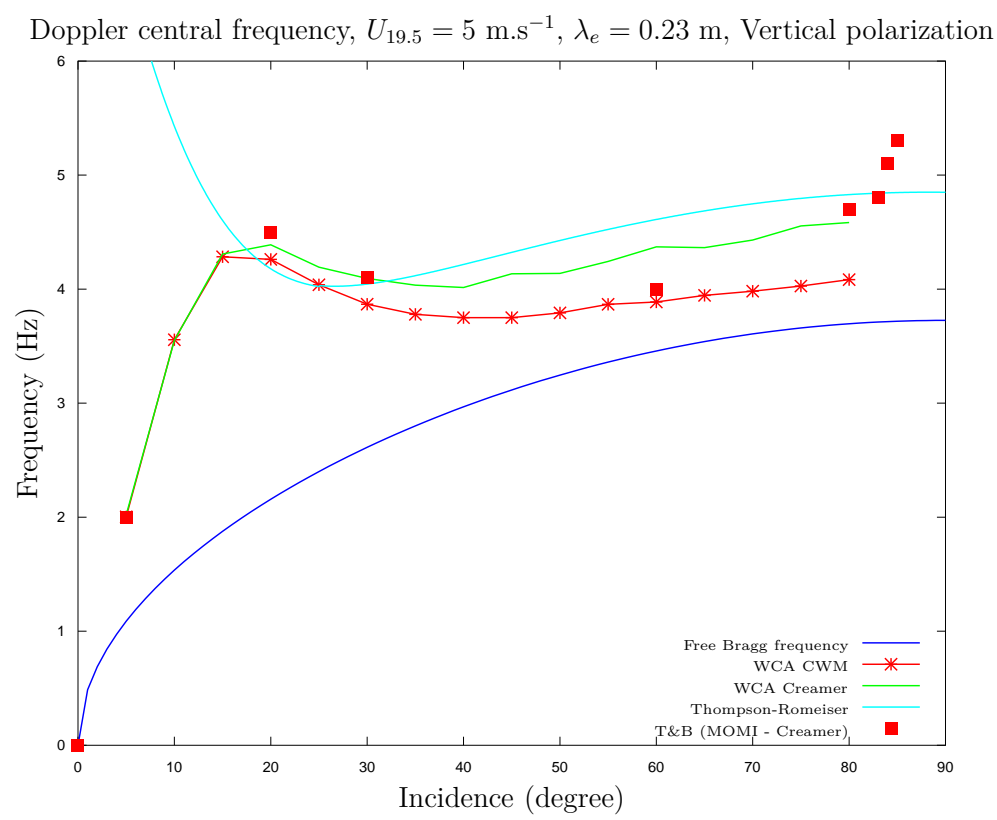

Fig. 6. Comparison of Monte-Carlo CWM and Creamer WCA Doppler central frequency in L band, vertical polarization with $U_{19.5}=5 \mathrm{~m} . \mathrm{s}^{-1}$. T\&B : Toporkov \& Brown results based on the MOMI and Creamer model. Thompson-Romeiser results are based on a TSM coupled with a MTF. 


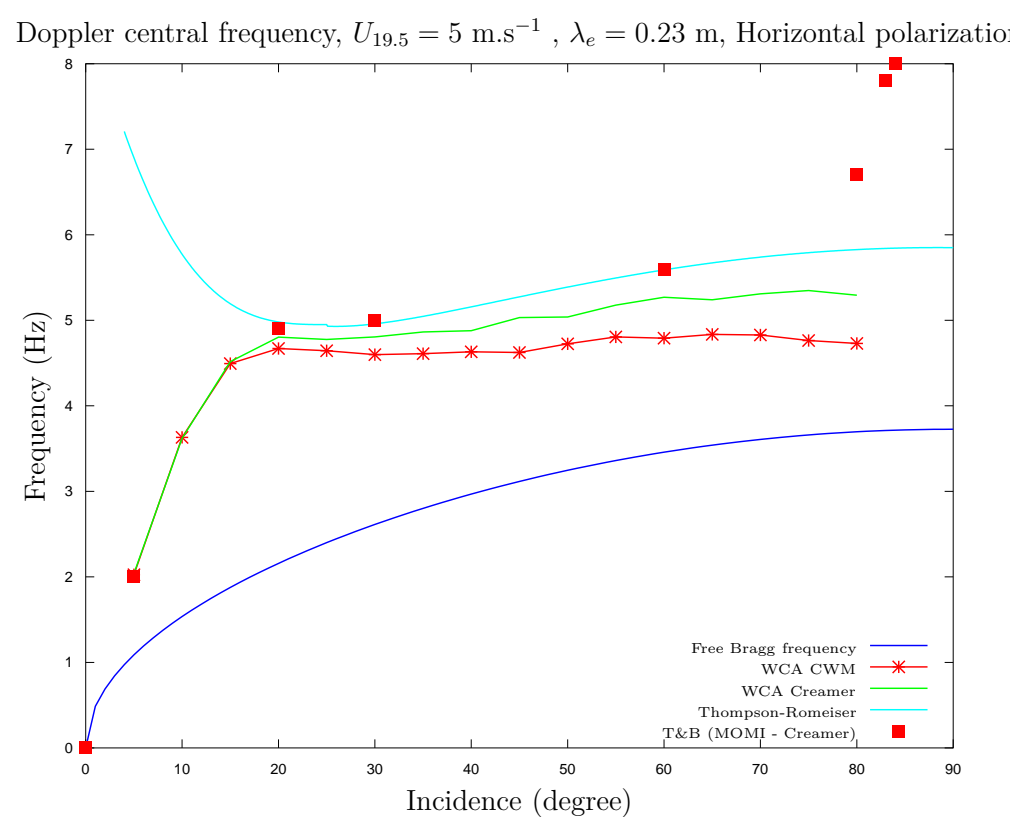

Fig. 7. Comparison of Monte-Carlo CWM and Creamer WCA Doppler central frequency in L band, horizontal polarization with $U_{19.5}=5 \mathrm{~m} . \mathrm{s}^{-1}$. T\&B : Toporkov \& Brown results based on the MOMI and Creamer model. Thompson-Romeiser results are based on a TSM coupled with a MTF.

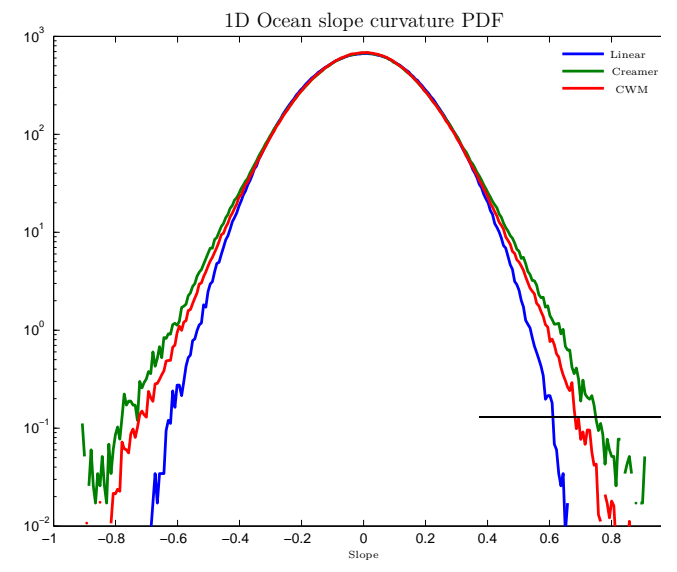

(a) Slope distribution

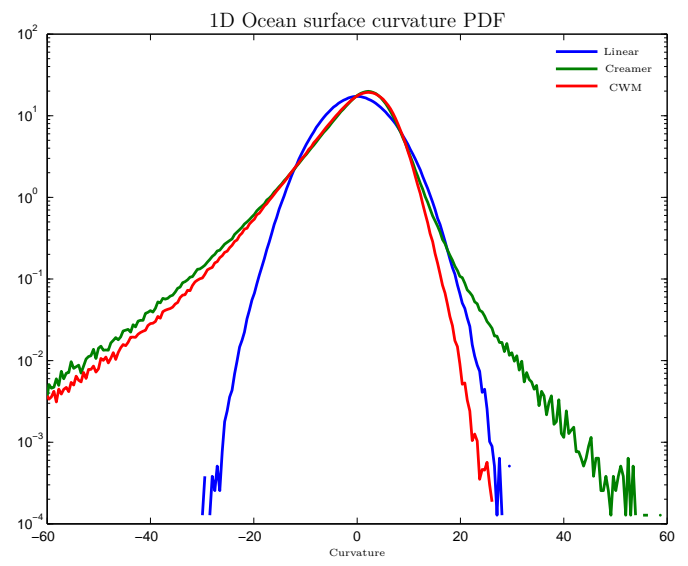

(b) Curvature disrtibution

Fig. 8. Probability distribution functions with a truncated Pierson Moskovitz spectrum at $k_{\max }=137 \mathrm{rad} . \mathrm{m}^{-1}, U_{19.5}=$ $5 \mathrm{~m} \cdot \mathrm{s}^{-1}$. 